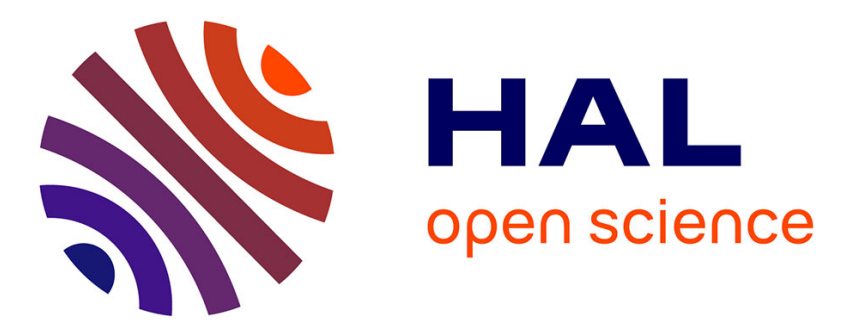

\title{
Characteristics of solution gated field effect transistors on the basis of epitaxial graphene on silicon carbide
}

Jürgen Ristein, Wenying Zhang, Florian Speck, Markus Ostler, Lothar Ley, Thomas Seyller

\section{- To cite this version:}

Jürgen Ristein, Wenying Zhang, Florian Speck, Markus Ostler, Lothar Ley, et al.. Characteristics of solution gated field effect transistors on the basis of epitaxial graphene on silicon carbide. Journal of Physics D: Applied Physics, 2010, 43 (34), pp.345303. 10.1088/0022-3727/43/34/345303 . hal00569687

\section{HAL Id: hal-00569687 \\ https://hal.science/hal-00569687}

Submitted on 25 Feb 2011

HAL is a multi-disciplinary open access archive for the deposit and dissemination of scientific research documents, whether they are published or not. The documents may come from teaching and research institutions in France or abroad, or from public or private research centers.
L'archive ouverte pluridisciplinaire HAL, est destinée au dépôt et à la diffusion de documents scientifiques de niveau recherche, publiés ou non, émanant des établissements d'enseignement et de recherche français ou étrangers, des laboratoires publics ou privés. 


\title{
Characteristics of solution gated field effect transistors on the basis of epitaxial graphene on silicon carbide
}

\author{
Jürgen Ristein, Wenying Zhang, Florian Speck, Markus Ostler, Lothar Ley and \\ Thomas Seyller \\ Lehrstuhl für Technische Physik, Friedrich-Alexander-Universität Erlangen-Nürnberg, \\ Erwin-Rommel-Str. 1, 91058 Erlangen, Germany
}

\begin{abstract}
A solution gated field effect transistor (SGFET) has been fabricated on epitaxial single layer graphene on $6 \mathrm{H}-\mathrm{SiC}(0001)$. Output and transfer characteristics were systematically studied as a function of electrolyte $\mathrm{pH}$. The transfer characteristics of the device show a $\mathrm{pH}$ dependent shift of $19 \pm 1 \mathrm{mV} / \mathrm{pH}$. From the minimum sheet conductivity observed, an average charge carrier mobility of $1800 \pm 100 \mathrm{~cm}^{2} \mathrm{~V}^{-1} \mathrm{~s}^{-1}$ at room temperature has been inferred. It turns out that the Fermi level in the graphene layer is strongly pinned in the vicinity of the Dirac point. The analysis of the transfer characteristics is consistent with a concentration of $4 \cdot 10^{14} \mathrm{~cm}^{-2}$ interface states at $0.1 \mathrm{eV}$ below the Dirac energy that is presumably associated with the $(6 \sqrt{ } 3 \times 6 \sqrt{ } 3) \mathrm{R} 30^{\circ}$ reconstruction at the interface between $\mathrm{SiC}(0001)$ and graphene.
\end{abstract}

PACS: $72.80 . \mathrm{Vp}, 73.20 .-\mathrm{r}, 73.40 . \mathrm{Mr}$

\begin{abstract}
1. Introduction
Graphene, the fifth allotrope of carbon, is a monolayer of carbon atoms arranged in a honeycomb structure. It is a gapless semiconductor with a six fold degenerate, point-like Fermi surface in the undoped case. The Fermi surface is defined by the intersection of linearly dispersing $\pi$-states that cross at the socalled Dirac point. The linear dispersion which corresponds to massless quasiparticles of electron and hole character and a chiral wave function for these states give rise to a number of exotic quantum effects that are not normally expected in semiconductors [1]. From a more mundane point of view, graphene is a semiconductor that is perfectly symmetrical in its electron and hole properties. Electron and hole concentrations can be varied over a wide range from about $10^{11}$ to $10^{14} \mathrm{~cm}^{-2}$ by applying appropriate fields across a dielectric [2] or by transfer doping from adsorbates such as F4-TCNQ [3]. On account of the reduced dimensionality and the chiral nature of the wavefunctions the scattering rates of carriers are reduced compared to conventional semiconductors and mobilities as high as $200,000 \mathrm{~cm}^{2} \mathrm{~V}^{-1} \mathrm{~s}^{-1}$ have been achieved at room temperature [4]. As such, graphene constitutes the ultimate two-dimensional (2d) electronic system. Unlike other $2 \mathrm{~d}$ systems such as the accumulation layers at semiconductor heterostructure interfaces or below the gate of MOS-devices, however, the carriers in graphene are directly exposed to the environment without any intervening layers. That should make graphene the ultimate sensor material and the transfer doping experiments alluded to above are among the first steps in this direction. Other venues have been explored as well [5].
\end{abstract}


However, the direct exposure to gases or adsorbates is but one possibility. A more fecund opportunity might lie in the so-called solution gate field effect transistor (SGFET). Here, graphene is immersed into an electrolyte and the carrier concentration is controlled by ionic charges that accumulate at or near the graphene-electrolyte interface. Such devices have been realized on the basis of Silicon MOSFETs where the gate electrode is replaced by an electrolyte [6]. More recently and closer to the case at hand, the p-type surface conductivity of hydrogenated diamond has been shown to be effectively controlled by ions when placed in an electrolyte [7],[8] By proper functionalisation of the diamond surface a variety of chemical and even biological sensors have been demonstrated in this way [9]. For graphene, where electrons as well as holes can contribute to the current an even wider field of applications as SGFET is to be expected provided the necessary functionalisation can be realized, a task that is helped considerably by the impressive body of experience with the functionalisation of carbon nanotubes.

In this paper we analyze $\mathrm{pH}$ dependent output and transfer characteristics of graphene-based SGFETs and compare our results with published work [10]. We also present the full potential diagram of the control loop including the $\mathrm{SiC}$ substrate on which graphene was grown and all contacts. In this way, the influence of substrate space charges and in particular of interface states between graphene and $\mathrm{SiC}$ has been taken into account. These defects are shown to have a decisive influence on the transfer characteristics for graphene on $\mathrm{SiC}$ and can therefore not be neglected.

\section{Experiment and Results}

The samples used in this experiment were graphene sheets produced on the silicon-terminated (0001) face of a semi-insulating, hexagonal $6 \mathrm{H}-\mathrm{SiC}$ single crystal by high-temperature annealing in an inert gas atmosphere [11]. The average thickness of the graphene layer was $1.05 \pm 0.1$ monolayers as determined from the x-ray excited photoelectron spectrum of the $\mathrm{C} 1 \mathrm{~s}$ core level by a procedure described elsewhere [12] with the inelastic mean free path adjusted such that consistency was reached with data obtained from low-energy electron microscopy [11]. Solution gated field effect transistors were produced on these templates by a procedure that was identically used in the past for hydrogen-terminated intrinsic diamond, and also the electrolyte and the experimental set up were chosen like in that study [7]. In the interest of the reader we describe these details here again: A U-shaped conductive graphene channel of dimension $14 \mathrm{~mm} \times 1.4 \mathrm{~mm}$ was defined by masking the channel area with a common photolithographic resist and removing the remainder of the graphene in an oxygen plasma (see figure 1 (a)). The channel was contacted at its ends by silver paste which yields perfectly ohmic contacts that serve as source and drain. By dipping the bottom part of the sample into solution the graphene channel is controlled over an effective length of $7.5 \mathrm{~mm}$ by the electrolytic gate. The part of the graphene channel outside the electrolyte shows a comparatively high sheet conductivity due to n-type doping which is commonly observed with graphene on $\mathrm{SiC}(0001)$ [13],[14],[15] and can for this experiment be simply regarded as an extension of the source and drain contacts.

The electrolyte consists of a $10 \mathrm{mM}$ phosphate buffer and $10 \mathrm{mM} \mathrm{KCl}$ which was titrated with $\mathrm{H}_{3} \mathrm{PO}_{4}$ and $\mathrm{KOH}$ to adjust the $\mathrm{pH}$. All chemicals were purchased from Roth (Germany) and were used without further purification. The gate electrode is provided by an electrochemical $\mathrm{Ag} / \mathrm{AgCl}$ reference electrode $(3 \mathrm{M} \mathrm{KCl}$ solution). As such we have chosen a double junction version (Schott B2220) to minimize contamination of the electrolyte. The red-ox potential of that electrode is $+0.20 \mathrm{~V}$ vs. standard hydrogen electrode (SHE) at room temperature. The graphene channel was grounded at the source contact and the gate voltage was applied to the gate electrode via a programmable voltage source. We have limited the range of operation with respect to drain-source and gate-source voltage such that the current between graphene and the gate electrode never exceeded $1 \mathrm{nA}$. In previous experiments we have observed an irreversible degradation of the graphene layer when such a provision was not taken. The drain-source-current was measured with a Keithley 617 electrometer. We use the sign convention for the voltages as illustrated in the schematic electrical circuit of figure 1b) where all voltages are referenced to ground. This corresponds to the common use in solid state electronics referring gate voltages to the source, but is opposite to the electrochemical convention referring working electrode potentials to the reference electrode. 
In figure 2 the field effect output characteristics are shown exemplary for $\mathrm{pH} 7$. For each gate-source voltage, which was varied between $-1.1 \mathrm{~V}$ and $+0.2 \mathrm{~V}$ in steps of $0.1 \mathrm{~V}$, we measure perfectly linear I-V characteristics up to the maximum drain-source voltage of $\left|\mathrm{V}_{\mathrm{ds}}\right|=0.1 \mathrm{~V}$. For a gate-source voltage of $-0.5 \mathrm{~V}$ a minimum in the channel conductance is observed, consistent with ambipolar transport of electrons and holes in the graphene channel. For the further analysis of our data we will define a surface potential $\Phi$ of the graphene channel (position $\mathrm{x}_{4}$ in the circuit diagram in figure 6) as the energy of the Dirac point (D) relative to the Fermi level $\left(\mathrm{E}_{\mathrm{F}}\right)$. Assuming identical mobilities of electrons and holes, the voltage of minimum conductivity obviously belongs to surface potential zero, and the corresponding gatesource voltage shall be denoted as the Dirac voltage $\mathrm{V}_{\text {Dirac }}(\mathrm{vs} . \mathrm{Ag} / \mathrm{AgCl})$ in the following.

Fixing the drain-source voltage at $\mathrm{V}_{\mathrm{DS}}=-50 \mathrm{mV}$, we have measured the transfer characteristics of the graphene $S G F E T$, i.e. the drain-source current $\mathrm{I}_{\mathrm{DS}}$ as a function of gate-source voltage $\mathrm{V}_{\mathrm{GS}}$, for different $\mathrm{pH}$ of the electrolyte as it is shown in figure 3. Except for extreme alkaline conditions ( $\mathrm{pH} 12)$, we find a universal minimum conductance of $6.8 \mu \mathrm{S}$ with a scatter of $3 \%$. Taking the aspect ratio of 5.2 (length over width) of the graphene channel into account this value corresponds to a minimum sheet conductivity of $(35 \pm 1) \mu \mathrm{S}$ which will be further discussed below. The transfer characteristics of figure 3 is systematically shifted with increasing $\mathrm{pH}$ to increasing gate-source voltages. On the anodic side (increasingly negative $\mathrm{V}_{\mathrm{GS}}$ ) this shift is almost ideally rigid, again with the exception of the most alkaline electrolyte, whereas on the cathodic side the I-V curves indicate a saturation that tends to be more pronounced for acidic electrolytes (compare also figure 5). We extract the $\mathrm{pH}$ sensitivity of the graphene SGFET and plot it in figure 4 as the voltage $\mathrm{V}_{\text {Dirac }}$ that belongs to minimum channel conductivity vs. $\mathrm{pH}$. We find a sensitivity factor of $19 \pm 1 \mathrm{mV} / \mathrm{pH}$ that is within the experimental uncertainty identical to the $\mathrm{pH}$ sensitivity that was found for SGFET's based on hydrogen-terminated and surface conductive diamond [7]. In the latter case, experiments on partially oxidized or aminated surfaces indicate that surface functional groups which are protonated with increasing and de-protonated with decreasing acidity of the electrolyte are responsible for the observed $\mathrm{pH}$ sensitivity [16]. More systematic studies on graphene are mandatory to identify the mechanism behind the $\mathrm{pH}$ sensitivity of the surface potential in the present case. At this point we may just note the striking similarity between the two carbon surfaces, despite their different electronic and structural properties.

$\mathrm{pH}$ dependent transfer characteristics for SGFET's based on single, double, and triple layer graphene have been reported before by Ang et al.. These authors do not present output characteristics of their devices. The most striking difference between their transfer characteristics for the single layer graphene case and our data is an apparently tenfold higher channel conductivity $\left(3.5 \cdot 10^{-4} \mathrm{~S}\right.$ in their work compared to $3.5 \cdot 10^{-5} \mathrm{~S}$ observed here for the Dirac voltage applied to the gate). Their channel conductivity is, however, much less efficiently controllable than in our case. We can roughly double the conductance with a gate voltage shift of $0.3 \mathrm{eV}$, whereas the same modulation in Ang et al. results only in an increase by $6 \%$. Nevertheless, in the linear range of the transfer characteristics, the slopes in absolute values are virtually the same $\left(\frac{\mathrm{d} \sigma_{\square}}{\mathrm{d} V_{G S}}=7 \cdot 10^{-5} \mathrm{~A} / \mathrm{V}^{2}\right.$ in [10] and about $\frac{\mathrm{d} \sigma_{\square}}{\mathrm{d} V_{G S}}=6 \cdot 10^{-5} \mathrm{~A} / \mathrm{V}^{2}$ in our data set). These features point to a large parasitic contribution to the drain-source current that is presumably due to a corresponding gate current in [10]. During our own experiments we have in fact observed an irreversible increase of gate currents when the graphene surface was polarized cathodically by more than $0.3 \mathrm{~V}$ vs. $\mathrm{Ag} / \mathrm{AgCl}$. This polarization corresponds to $\mathrm{V}_{\mathrm{GS}}=+0.3 \mathrm{~V}$ in our and Ang's notation. (In both works a $\mathrm{Ag} / \mathrm{AgCl}$ reference electrode was used.) Beyond this voltage defects are created in the graphene layer, and a subsequent photoemission study even indicates that holes were formed in the graphene layer. We have limited the gate voltage range accordingly in our experiment in order avoid the creation of surface defects. In the cited work by Ang et al., however, cathodic polarization up to $1.0 \mathrm{~V}$ was adopted, which most likely created a large defect density that explains the high parasitic currents across the graphene layer. This conclusion is corroborated by a very strong and in fact super-Nernstian $\mathrm{pH}$ response of $98 \mathrm{mV} / \mathrm{pH}$ observed by Ang et al strongly indicating a large concentration of surface defect sites for proton and/or 
hydroxyl adsorption. This reasoning is further supported by a critical review of the analysis of the electronic characteristics presented by Ang et al. as will be discussed in the next paragraph.

\section{Discussion}

For further evaluating our data, we have re-plotted figure 3 in figure 5 with the gate voltage relative to the Dirac voltage and with the ordinate normalized to the minimum sheet conductivity. The master curve obtained in this way is nearly independent on $\mathrm{pH}$ on the anodic side (except for $\mathrm{pH} 12$ ) and shall be discussed in the following.

To this end we consider the full gate-source circuit including the solid state and the electrolytic part in the band diagram shown in figure 6 . The potential shown is the electrostatic energy of a negative unit charge in the circuit. For convenience, we tied this potential in graphene to the Dirac energy D. The Dirac energy is experimentally found to be $0.7 \mathrm{eV}$ below the conduction band minimum [17],[15] $\mathrm{E}_{\mathrm{C}}$ of $\mathrm{SiC}$ which fixes the band edges $\mathrm{E}_{\mathrm{C}}$ and $\mathrm{E}_{\mathrm{V}}$ of the substrate in figure 6. Coordinates $\mathrm{x}_{1}$ to $\mathrm{x}_{8}$ mark the corresponding positions in the circuit diagram in Fig 1(b). The scale in this circuit diagram covers nm sections such as the metal-graphene interface, the graphene layer cross sections $\left(\mathrm{x}_{4}\right)$, and the compact part of the electrolytic double layer at the graphene electrolyte interface; $\mu \mathrm{m}$ sections such as the space charge regions in the silicon carbide substrate and the diffuse layer of the electrolyte; and $\mathrm{mm}$ regions covering the lateral path in the $\mathrm{SiC}$ from the contact range to the gate electrode range of the graphene overlayer $\left(\mathrm{x}_{2}\right.$ to $\mathrm{x}_{3}$ ) and in the electrolyte between the diffuse layer and the gate electrode. In the macroscopic parts of the circuit the half cell potential $\mathrm{eG}_{\mathrm{REF}}$ of the $\mathrm{Ag} / \mathrm{AgCl}$ reference electrode and the gate potential $\mathrm{eV}_{\mathrm{GS}}$ are schematically indicated as discontinuities. The contact potential between graphene and the metal contact is not shown explicitly; it has a constant value and is part of the total Galvani potential between the bulk SiC substrate and the contact metal. The semi-insulating $\mathrm{SiC}$ is sketched as intrinsic with a charge neutrality level $\mathrm{E}_{0}$ close to mid gap. The work function of the source contact metal is exemplarily chosen smaller than that of $\mathrm{SiC}$ and smaller than the electron affinity of graphene so that it is electron-injecting. These features which were chosen in a plausible but arbitrary manner have no influence on our data analysis. By choosing the circuit path in figure 1(b) through the bulk of $\mathrm{SiC}\left(\mathrm{x}_{2}, \mathrm{x}_{3}\right)$ the potential profile between bulk $\mathrm{SiC}$ and the source metal contact is constant and can thus be neglected further on. Also the voltage drop across graphene $\left(\mathrm{x}_{4}\right)$ can be neglected compared to the voltage drops across the Helmholtz layer and the space charge region in $\mathrm{SiC}$. Consequently, we have assigned a constant potential across the graphene layer as indicated by the horizontal lines. Ionic charges are marked with circles in figure 6, charges in the solid state sections just by + and - signs. In the solid state sections the Fermi level is indicated as a dashed line. The potential is chosen to be zero asymptotically in the bulk electrolyte. Corresponding to Kirchhoffs loop rule of electrostatics the total potential drop across the circuit must be zero.

We will first qualitatively discuss the voltage shift of the transfer characteristics with varying $\mathrm{pH}$. To this end we consider a point of fixed drain-source current on one branch of the $\mathrm{I}_{\mathrm{DS}} \mathrm{vs}$. $\mathrm{V}_{\mathrm{GS}}$ curve of figure 3 . Let us further assume that the band diagram of figure 6 is just referring to this situation. We will discuss what change of $\mathrm{V}_{\mathrm{GS}}$ is required to compensate the effect of say decreasing $\mathrm{pH}$, i.e. we will ask what change of $\mathrm{V}_{\mathrm{GS}}$ is needed to keep the drain-source current constant despite a change in electrolyte $\mathrm{pH}$. Since the channel conductivity shall be unchanged, the complete potential diagram between $\mathrm{x}_{1}$ and the inner Helmholtz plane (IHP) must be unchanged as well. Decreasing $\mathrm{pH}$, i.e. increasing acidity, will in general supply more hydronium $\left(\mathrm{H}_{3} \mathrm{O}^{+}\right)$and less hydroxile $\left(\mathrm{OH}^{-}\right)$ions to the graphene electrolyte interface. This will turn the density of adsorbed ionic charge at the IHP more positive by a certain amount $\sigma_{+}$resulting in the corresponding change $-\sigma_{+}$in the depth integrated charge density of the diffuse layer. The consequence for the potential in figure 6 is obviously a rise in the profile between IHP and $\mathrm{x}_{5}$. The potential at $\mathrm{x}_{5}$ is shifted up by $\Delta E=e \cdot \sigma_{+} / C$ where $\mathrm{C}$ is the combined areal capacitance of the outer compact layer (IHP to outer Helmholtz plane, OHP) and the diffuse layer (OHP to $\mathrm{x}_{5}$ ). $\mathrm{C}$ is usually dominated by the outer compact layer capacitance. Obviously, -e $\mathrm{V}_{\mathrm{GS}}$ must increase by the same value

$\Delta \mathrm{E}$ to keep Kirchhoff's loop rule valid for the potential diagram of figure 6 . Thus, increasing acidity (decreasing $\mathrm{pH}$ ) shifts the transfer characteristics to more negative values on the $\mathrm{V}_{\mathrm{GS}}$ scale, in accordance with figure 4. 
Assuming a constant charge carrier mobility for the electrons and holes in graphene, the sheet conductivity measured in figure 3 is proportional to the (areal) charge carrier density (electrons plus holes!) in the graphene channel as a function of the gate voltage $\mathrm{V}_{\mathrm{GS}}$. We consider the surface potential $\Phi$ as defined above (energy of the Dirac point in the graphene layer (at $\mathrm{x}_{4}$ ) relative to the Fermi level). The band diagram in figure 6 thus exemplarily refers to a positive surface potential. The areal excess electron density $\mathrm{n}$ in the graphene, i.e. in the $\pi^{*}$ band above the Dirac energy, is found as a function of surface potential $\Phi$ by integrating the Fermi-Dirac distribution function over the linearly increasing twodimensional density of states:

$$
n(\Phi)=\int_{0}^{\infty} \frac{\kappa \cdot E}{1+\exp \left(\frac{E+\Phi}{k T}\right)} \mathrm{d} E=\int_{\Phi}^{\infty} \kappa \cdot \frac{E-\Phi}{1+\exp \left(\frac{E}{k T}\right)} \mathrm{d} E
$$

where $\kappa=(1.18 \pm 0.06) \cdot 10^{14} \mathrm{~cm}^{-2}(\mathrm{eV})^{-2}$ is the slope of the two-dimensional density of states $D_{G}(E)=\kappa \cdot|E|$ of graphene [15]. $\kappa$ is linked via Planck's constant to the Fermi velocity $\mathrm{v}_{\mathrm{F}}$ by $\kappa=2 /\left(\pi \hbar^{2} \mathrm{v}_{\mathrm{F}}^{2}\right)$. Evaluating (1) yields (see appendix)

$$
\frac{n(x)}{\kappa \cdot(k T)^{2}}=\alpha+\left\{\begin{array}{c}
\frac{1}{2} x^{2}+\int_{0}^{-x} R(t) \mathrm{d} t \text { for } x \leq 0 \\
-\int_{0}^{x} R(t) \mathrm{d} t \text { for } x \geq 0
\end{array} .\right.
$$

Here, $x=\Phi /(k T)$ is the surface potential in units of the thermal energy $k T, n_{0}=\kappa \cdot(k T)^{2}$ a natural unit for the areal charge carrier concentration of a single graphene layer which amounts to $7.4 \cdot 10^{10} \mathrm{~cm}^{-2}$ at room temperature, and $\alpha=\int_{0}^{\infty} u \cdot[1+\exp (u)]^{-1} \mathrm{~d} u=\pi^{2} / 12=0,8225$.

$R(t)=\int_{t}^{\infty}[1+\exp (u)]^{-1} \mathrm{~d} u=\ln [1+\exp (-t)]$ is the Fermi residuum, for which $\exp (-t)$ is a strict upper limit that is approached asymptotically for positive argument. From the asymptotic limit required for equation (2), $\lim _{x \rightarrow \infty} n(x)=0$, it is obvious that $\alpha$ can alternatively be expressed as the complete integral of the Fermi residuum, i.e. $\alpha=\int_{0}^{\infty} \int_{t}^{\infty}[1+\exp (u)]^{-1} d u d t=\int_{0}^{\infty} x \cdot[1+\exp (x)]^{-1} d x$. This is also seen immediately by changing the order of integration of the left hand side double integral. Note that the zerotemperature limit for the electron statistics would just give the parabolic term in equation (2), specifically it would yield a zero conductivity contribution of the electrons with the Fermi level at the Dirac point.

From the symmetry of the graphene density of states and the Fermi function it is straight forward to conclude $p(x)=n(-x)$ for the hole concentration as a function of (normalized) surface potential. Combining this with equation (2) yields

$$
\frac{\Sigma_{g}(x)}{e \cdot \kappa \cdot(k T)^{2}}=\left[\frac{1}{2} x^{2}+2 \int_{0}^{|x|} R(t) \mathrm{d} t\right] \cdot \operatorname{sign}(x)
$$

for the areal charge density $\Sigma_{\mathrm{g}}$ in the graphene layer, and 


$$
\frac{c(x)}{\kappa \cdot(k T)^{2}}=2 \alpha+\frac{1}{2} x^{2}
$$

for the areal charge carrier concentration $\mathrm{c}=\mathrm{n}+\mathrm{p}$ in the graphene layer. Note that $\Sigma_{\mathrm{g}}(x)$ and $c(x)$ are odd and even functions of the surface potential, respectively. Assuming the same mobility $\mu$ for electrons and holes in graphene, equation (4) yields directly the sheet conductivity $\sigma_{\square}(x)=e \cdot \mu \cdot c(x)$ as a function of surface potential with $e$ being the elementary charge. Specifically, we find $\sigma_{\square, \min }=2 \alpha \cdot \kappa(k T)^{2} \cdot e \cdot \mu$ for the minimum sheet conductivity $(x=0)$. From our experiment we extract $\sigma_{\square, \min }=35 \pm 1 \mu \mathrm{S}$. This yields $1800 \pm 100 \mathrm{~cm}^{2} \mathrm{~V}^{-1} \mathrm{~s}^{-1}$ for the mobility of electrons and holes in the graphene layer of our SGFET when the Fermi level coincides with the Dirac energy.

With the further assumption of equal and constant electron and hole mobility we can infer

$$
\frac{\sigma_{\square}(x)}{\sigma_{\square, \min }}=1+\frac{x^{2}}{4 \alpha}
$$

for the normalized sheet conductivity that is plotted in figure 5. Note that the channel conductivity varies in our experiment by less than a factor of 2.2. which certainly justifies the assumption of a mobility independent of charge carrier concentration. Equation (5) is easily inverted and thus allows calculating the surface potential from the normalized sheet conductivity:

$$
x=\frac{\Phi}{k T}= \pm \sqrt{4 \alpha \cdot\left(\frac{\sigma_{\square}(x)}{\sigma_{\square, \min }}-1\right)}
$$

where the positive sign refers to the branch with negative relative gate voltage in figure 5 (hole excess) and vice versa. Using equation (6) we can transform the transfer characteristics to evaluate the surface potential in the graphene layer as a function of the relative gate-source voltage. This is plotted in figure 7. For the further discussion consider the inverse of the plot in figure 7, i.e. take the surface potential as a variable and construct the corresponding relative gate-source voltage. From figure 6 the surface potential $\Phi$ itself constitutes the voltage change (with respect to the $\Phi=0$ situation) across the section $\mathrm{x}_{1}$ to $\mathrm{x}_{4}$ of the circuit. The change of the voltage drop (again with respect to $\Phi=0$ ) across the Helmholtz layer is proportional to the charge modulation $\Sigma(\Phi)$ in the SiC space charge layer $\left(\mathrm{x}_{3}\right.$ to $\left.\mathrm{x}_{4}\right)$ plus the graphene layer $\left(\mathrm{x}_{4}\right)$, devided by the (areal) Helmholtz capacitance $\mathrm{C}_{\mathrm{HH}}$ :

$$
-e \cdot V_{G S}=\Phi+\frac{e}{C_{H H}} \cdot \Sigma(\Phi)
$$

Due to the high background ion concentration of the electrolyte used here $(3 \mathrm{M} \mathrm{KCl})$, the voltage drop across the diffuse layer in the electrolyte can be neglected, i.e. we can approximate the excess ionic charge in the diffuse layer of the electrolyte by a sheet charge at the OHP. For the areal capacitance of the compact layer we use $15 \mu \mathrm{F} / \mathrm{cm}^{2}$. This value corresponds to nominal thicknesses of $d_{1}=0.25$ and $d_{2}=0.40 \mathrm{~nm}$ and dielectric constants of $\varepsilon_{1}=6$ and $\varepsilon_{2}=25$ for the inner (surface to IHP) and outer (IHP to $\mathrm{OHP}$ ) part of the compact layer, repectively, and was found appropriate for the compact layer capacitance of graphite in previous work [18]. The integrated areal charge density $\Sigma(\Phi)$ is composed of three contributions: $\Sigma(\Phi)=\Sigma_{g}+\Sigma_{\text {def }}+\Sigma_{S i C}$. The first term corresponds to the areal charge density in the graphene layer as specified by equation (3). The second term takes defect states into account due to the 
reconstructed interface layer between graphene and the $\mathrm{SiC}$ substrate. This interface layer is the $(6 \sqrt{3} \times 6 \sqrt{3}) \mathrm{R} 30^{\circ}$ reconstruction of the $\mathrm{SiC}(0001)$ surface which has been shown to create a surface (or interface) state band with little dispersion and considerable density of states [19]. If we specify the twodimensional density of states of that band as $D_{\text {def }}(E)$ with $E$ taken relative to the Dirac energy of the graphene layer, the areal space charge accommodated in the interface defects can be written as

$$
\Sigma_{\text {def }}(\Phi)=-e \cdot \int_{-\infty}^{\infty} \frac{D_{\text {def }}(E)}{1+\exp \left(\frac{E+\Phi}{k T}\right)} \mathrm{d} E+e \cdot N_{+}
$$

where $e N_{+}$is the hypothetical areal charge density if the defect band were completely unoccupied. Finally, the third contribution $\Sigma_{\mathrm{SiC}}$ represents the charge in the space charge layer of the $\mathrm{SiC}$ substrate. Since semi-insulating $\mathrm{SiC}$ material has been used, it can easily be shown that $\Sigma_{\mathrm{SiC}}$ is negligible when compared to $\Sigma_{\mathrm{g}}$ and $\Sigma_{\text {def }}$ for all possible scenarios of band bending. Inserting equations (3) and (8) into (7) and forming the derivative yields

$$
-\frac{\mathrm{d}\left(e V_{G S}\right)}{\mathrm{d} \Phi}=1+\frac{e^{2}}{C_{\mathrm{HH}}}\left[\kappa|\Phi|+2 \kappa(k T) \cdot R\left(\left|\frac{\Phi}{k T}\right|\right)+\left\{D_{\mathrm{def}} * g\right\}(-\Phi)\right] .
$$

The last term in the brackets on the right hand side is the convolution of the defect density of states with the negative derivative of the Fermi Dirac function, taken at energy $-\Phi$ (relative to the Dirac energy), i.e. $g(E)=-\frac{\mathrm{d}}{\mathrm{d} E}\left[\frac{1}{1+\exp \left(\frac{E}{k T}\right)}\right]=\frac{1}{k T} \frac{\exp \left(\frac{E}{k T}\right)}{\left(1+\exp \left(\frac{E}{k T}\right)\right)^{2}}$, which is an even function in its argument with area 1. Thus, $S(E)=\left\{D_{\text {def }} * g\right\}(E)$ is a spectroscopic defect density of states function for the SiC/graphene interface, probed with $g(E)$ as resolution function. $S(\Phi)$ is easily obtained from inverting $(9)$ :

$$
S(-\Phi)=D_{0} \cdot\left\{\left[-\frac{d\left(e V_{\mathrm{GS}}\right)}{d \Phi}-1\right] \cdot \frac{C_{\mathrm{HH}}}{C_{0}}-\left|\frac{\Phi}{k T}\right|-2 \cdot R\left(\left|\frac{\Phi}{k T}\right|\right)\right\}
$$

where $D_{0}=\kappa k T=2.9 \cdot 10^{12} \mathrm{~cm}^{-2}(\mathrm{eV})^{-1}$ at room temperature. $C_{0}=e^{2} \kappa k T$ is a constant that amounts to $0.47 \mu \mathrm{F} \mathrm{cm}^{-2}$ at room temperature and is thus small compared to the Helmholtz capacitance of $\mathrm{C}_{\mathrm{HH}}=15 \mu \mathrm{F} \mathrm{cm}^{-2}$ that has been introduced before. In fact, the second and third terms in the bracket on the right hand side of (10) are smaller than 2 within the variation of the surface potential $\Phi$ in our experiment and thus constitute only a minor correction to the first term which is essentially the derivative of the gate control voltage w.r.t. the surface potential. Nevertheless, we have taken them into account when transforming the data of figure 7 via eq. (10) into the spectroscopic defect density of states shown in figure 8 . The spectrum shows a minimum around the Dirac energy with steep increase towards higher energies (i.e. towards the $\mathrm{SiC}$ conduction band minimum) and lower energies (i.e. towards the $\mathrm{SiC}$ mid gap energy). We have also plotted the resolution function $g(E)$ for two cases, centred at $-100 \mathrm{meV}$ (dashed line) and centred at $-70 \mathrm{meV}$ (dash-dotted line) relative to the Dirac energy, respectively. In both cases, the tail of the resolution function fits the spectroscopic DOS. Thus, the data are consistent with a narrow (in fact $\delta$-function type) band of defect states whose areal density $\mathrm{n}_{\text {def }}$ is identical with the scaling factor of the resolution function fitted to the data. For case 1, this scaling factor is 
$11000 \kappa(k T)^{2}=8.1 \cdot 10^{14} \mathrm{~cm}^{-2}$ and for case 2 it is $4000 \kappa(k T)^{2}=3.2 \cdot 10^{14} \mathrm{~cm}^{-2}$. The data are thus consistent with a band of defect states between 70 and $100 \mathrm{meV}$ below the Dirac energy and with concentration between 3.2 and $8.1 \cdot 10^{14} \mathrm{~cm}^{-2}$. This latter upper limit is, however, not unique due to the exponential character of the resolution function for sufficiently large argument. The data are thus equally consistent with any $A$-fold larger concentration of defects shifted by $k T \cdot \ln (A)$ further towards lower energies into the band gap of SiC. However, $8.1 \cdot 10^{14} \mathrm{~cm}^{-2}$ corresponds already to $65 \%$ of the surface atom density of the $\mathrm{SiC}(0001)$ surface $\left(1.22 \cdot 10^{15} \mathrm{~cm}^{-2}\right)$, and to about $20 \%$ of the graphene atom density. Thus, two out of three Si interface atoms would be expected to give rise to a dangling bond-like defect in case 1 . A substantially larger defect density than that is hard to imagine. We may therefore consider the range of energies and concentrations specified above as a reasonable estimate for the defect DOS.

On the high energy side of figure 8 the data show a strong scatter and the spectrum increases even stronger than the resolution function allows. We may speculate that here already disorder induced tail states of the conduction band of the $\mathrm{SiC}$ become visible whose density of states is expected to increase exponentially to higher energies. We have to admit at this point, that the data allow no further conclusions.

Finally, we would like to reconsider equations (3) and (4) for the areal charge and charge carrier density, respectively, of graphene as a function of temperature and surface potential. They constitute fundamental relationships for the analysis of conductivity and capacitance experiments on graphene based devices. In fact, in the absence of interface defects and when neglecting the space charge in the substrate onto which the graphene layer is prepared, equation (4) directly yields the temperature dependent charge carrier concentration and thus the sheet conductivity with the charge carrier mobility as the connecting parameter. For small variation of the surface potential around zero as in our experiment, the assumption of a mobility independent of the charge carrier density in graphene is certainly justified. Equation (4) is, however, generally valid as long as the dispersion relation of the $\pi$ and the $\pi^{*}$ bands remain linear and the occupation of the $\sigma$ and $\sigma^{*}$ bands with holes and electrons, respectively, can be neglected. Moreover, equation (3) yields the temperature dependent areal differential capacitance $C_{g}$ of a graphene layer as

$$
C_{\mathrm{g}}(\Phi, T)=e \frac{\mathrm{d} \Sigma_{g}}{\mathrm{~d} \Phi}=e^{2} \kappa \cdot k T \cdot\left[2 R\left(\frac{\Phi}{k T}\right)+\frac{\Phi}{k T}\right]
$$

The prefactor $C_{0}=e^{2} \kappa \cdot k T$ was already defined above. For $\Phi=0$, equation (11) gives a minimum areal differential capacitance $C_{\min }(T)=2 \ln (2) \cdot C_{0}(T)$ that amounts to $0.66 \mu \mathrm{F} \mathrm{cm}^{-2}$ at room temperature. This value is in excellent agreement with experimental data [20]. We note in passing that this finite minimum areal capacitance is an intrinsic property of graphene and does not require charge inhomogeneities as suggested in [20].

We have plotted the areal charge carrier concentration $c(\Phi, T)$ corresponding to (3) and the areal differential capacitance $C_{\mathrm{g}}(\Phi, T)$ corresponding to (11) in figure 9. The temperature dependence is in both cases taken into account in form of the natural units adopted for the left hand side ordinates and the lower abscissa. The right hand scale and the upper abscissa refer to the specific quantities for room temperature. An equivalent albeit more unhandy expression for $\mathrm{C}_{\mathrm{g}}$ was derived before by Fang et al. and named 'quantum capacitance' for graphene. This notation is misleading since there is no quantum effect involved here. On the contrary, the concept of a constant and temperature independent areal differential capacitance can in fact be defined only for conventional unipolar $2 \mathrm{~d}$ charge carrier gases as $C_{\mathrm{q}}=n \cdot 4 \pi \mathrm{m}^{*} e^{2} / \hbar^{2}$ where $\mathrm{n}$ is an integer band filling factor and $\mathrm{m} *$ the effective carrier mass [21]. It is obvious that this concept is meaningless for the massless Dirac particles in graphene. The misconception of a quantum capacitance (in the sense of being universal and constant) for graphene might have caused some confusion also in the analysis of Ang et al. [10] of their data on graphene based SGFET's. By simply 
setting such a quantum capacitance to $0.020 \mu \mathrm{F} \mathrm{cm}^{-2}$ without traceable justification they derive electron and hole mobilities of $1800 \mathrm{~cm}^{2} \mathrm{~V}^{-1} \mathrm{~s}^{-1}$ and $3100 \mathrm{~cm}^{2} \mathrm{~V}^{-1} \mathrm{~s}^{-1}$, respectively, from the slope in the linear range of their transfer characteristics. Besides sounding plausible, these values have no scientific foundation. Needless to say that interface defects, that have in our work been identified to play a key role for the field effect in epitaxial graphene, are completely ignored in Ang et al. [10].

\section{Conclusion}

In summary, we have presented output and transfer characteristics of SGFETs based on epitaxial single layer graphene on semi-insulating $6 \mathrm{H} \mathrm{SiC}(0001)$, carefully limiting the maximum polarisation of the graphene layer vs. $\mathrm{Ag} / \mathrm{AgCl}$ such that defect creation is excluded. We find a $\mathrm{pH}$ sensitivity of $19 \mathrm{meV} / \mathrm{pH}$ that is identical to the one found on hydrogen-terminated diamond, probably a coincidence. The quantitative analysis of the data shows that the Fermi level varies by no more than $\pm 50 \mathrm{meV}$ around the

Dirac energy due to strong pinning by interface states of the $(6 \sqrt{ } 3 \times 6 \sqrt{ } 3) \mathrm{R} 30^{\circ}$ reconstruction of the $\mathrm{SiC}$ (0001) substrate.

\section{Acknowledgement}

This work was financially supported by the German Research Foundation (DFG) within project SE1087/5-1 and within the framework of the Cluster of Excellence 'Engineering of Advanced Materials' at the University of Erlangen-Nuremberg. One of us (W.Z.) was supported by the DFG within the framework of the Research Training Group 1161 'Disperse Systeme für Elektronikanwendungen'.

\section{References}

[1] Novoselov K S, Geim A K, Morozov S V, Jiang D, Katsnelson M I, Grigorieva I V and Dubonos S V 2005 Nature 438197

Zhang Y B, Tan Y W, Störmer H L and Kim P 2005 Nature 438201

[2] Novoselov K S, Geim A K, Morozov S V, Jiang D, Zhang Y, Dubonos S V, Grigorieva I V and Firsov A A 2004 Science 306666

[3] Chen W, Chen S, Qi D C, Gao X Y and Wee A T S 2007 J. Am. Chem. Soc. 12910418

[4] Orlita M et al. 2008 Physical Review Letters 101267601

Bolotin K I, Sikes K J, Jiang Z, Klima M, Fudenberg G, Hone J, Kim P and Störmer H L 2008 Solid State Communications 146351

[5] Schedin F, Geim A K, Morozov S V, Hill E W, Blake P, Katsnelson M I and Novoselov K S 2007 Nat. Mater. 6652

[6] Bergveld P 2003 Sensors and Actuators B: Chemical 881

[7] Ristein J, Zhang W and Ley L 2008 Phys. Rev. E 78041602

[8] Zhang W, Ristein J and Ley L 2008 Phys. Rev. E 78041603

[9] Nebel C E, Rezek B, Shin D, Uetsuka H and Yang N 2007 J. Phys. D: App. Phys. 406443

[10] Ang P K, Chen W C, Wee A T S and Loh K P 2008 J. Am. Chem. Soc. 13014392

[11] Emtsev K V et al. 2009 Nature Materials 8203

[12] Seyller T H et al. 2006 Surface Science 6003906

[13] Berger C et al. 2006 Science 3121191

[14] Emtsev K V, Seyller Th, Speck F, Ley L, Stojanov P, Riley J D, Leckey R G C 2007 Mater. Sci. Forum 556-557 525

[15] Ohta T, Bostwick A,. McChesney J L, Seyller T, Horn K and Rotenberg E 2007 Phys. Rev. Lett. 98, 206802

[16] Song K S, Nakamura Y, Sasaki Y et al. 2006 Anal.Chim. Acta 5733.

[17] Seyller T H, Emtsev K V, Speck F, Gao K Y and LeyL 2006 Appl. Phys. Lett. 88242103

[18] Gerischer H, McIntiyre R, Scherson D and Storck W 1987 J. Phys. Chem. 91, 1930 
[19] Emtsev K V Speck F, Seyller Th, Riley J D and Ley L 2008 Phys. Rev. B 77, 155303

[20] Chen Z and Appenzeller J 2008 IEEE IEDM Technical Digest 21.1, 509

[21] John D L, Castro L C and Pulfrey D L 2004 J. App. Phys. 96, 5180

\section{Appendix. Evaluation of electron and hole densities for graphene at finite temperature}

We follow an approach taken before by Gerischer et al. for the analysis of capacitance data on graphite [18] and adopt it for the case at hand.

We start with the general expression for the areal or volume density of electrons in a system with two or three dimensional density of states $D(E)$ which can be separated in three parts due to the symmetry of the Fermi-Dirac function $f(E)=[1+\exp (\beta E)]^{-1}$ :

$$
\int_{-\infty}^{\infty} D(E) \cdot f\left(E-E_{F}\right) \mathrm{d} E=\int_{-\infty}^{E_{F}} D(E) \mathrm{d} E+\int_{E_{F}}^{\infty} D(E) \cdot f\left(E-E_{F}\right) \mathrm{d} E-\int_{-\infty}^{E_{F}} D(E) \cdot f\left(E_{F}-E\right) \mathrm{d} E .
$$

We will evaluate this for the areal electron density $n(\Phi, T)$ in graphene by choosing the origin of the energy axis to be the Fermi energy and by substituting $D(E)=\kappa \cdot(E-\Phi) \cdot \Theta(E-\Phi)$ with the surface potential $\Phi$ as defined in the text and the Heavyside function $\Theta(E)$. We will separately discuss negative and positive sign of $\Phi$.

Case $1, \Phi \leq 0$ :

The first term on the left hand side of (A1) gives the trivial, temperature independent result $n_{1}=\frac{1}{2} \kappa \Phi^{2}$. The second term gives

$$
n_{2}=\int_{0}^{\infty} \kappa \cdot(E-\Phi) \cdot[1+\exp (\beta E)]^{-1} \mathrm{~d} E
$$

and the third term on the right hand side can be written as

$$
\begin{aligned}
n_{3}=-\int_{\Phi}^{0} \kappa \cdot(E & -\Phi) \cdot[1+\exp (-\beta E)]^{-1} \mathrm{~d} E=\int_{-\Phi}^{0} \kappa \cdot(-E-\Phi) \cdot[1+\exp (\beta E)]^{-1} \mathrm{~d} E \\
& =\int_{0}^{-\Phi} \kappa \cdot(E+\Phi) \cdot[1+\exp (\beta E)]^{-1} \mathrm{~d} E \\
& =\int_{0}^{\infty} \kappa \cdot(E+\Phi) \cdot[1+\exp (\beta E)]^{-1} \mathrm{~d} E-\int_{-\Phi}^{\infty} \kappa \cdot(E+\Phi) \cdot[1+\exp (\beta E)]^{-1} \mathrm{~d} E
\end{aligned}
$$

Summing up $n_{2}$ and $n_{3}$ gives 


$$
\begin{aligned}
n_{2}+n_{3}= & 2 \kappa \cdot \int_{0}^{\infty} E \cdot[1+\exp (-\beta E)]^{-1} \mathrm{~d} E \\
& -\frac{\kappa}{\beta^{2}} \cdot\left\{\int_{-\beta \Phi}^{\infty} t \cdot[1+\exp (t)]^{-1} \mathrm{~d} t+\beta \Phi \cdot \int_{-\beta \Phi}^{\infty}[1+\exp (t)]^{-1} \mathrm{~d} t\right\}
\end{aligned}
$$

The first term on the right-hand-side is simply evaluated giving $2 \alpha \cdot \kappa \cdot(k T)^{2}$ where the constant $\alpha=\pi^{2} / 12$ and $k T=1 / \beta$ for the thermal energy have been inserted. The second term on the r.h.s. is further evaluated by discussing the expression in brackets as a function $h(y)=h(-\beta \Phi)$. Forming the derivative of $\mathrm{h}$ gives

$$
\begin{aligned}
\frac{\mathrm{d} h}{\mathrm{~d} y} & =-\frac{y}{1+\exp (y)}-\left\{\int_{y}^{\infty}[1+\exp (t)]^{-1} \mathrm{~d} t-y \cdot[1+\exp (y)]^{-1}\right\} \\
& =-\int_{y}^{\infty}[1+\exp (t)]^{-1} \mathrm{~d} t=-R(y)
\end{aligned}
$$

From the definition of $h(y)$, the value for zero follows as $h(0)=\alpha$ so that $h(y)=\alpha-\int_{0}^{y} R(t) \mathrm{d} t$, and the electron density $n_{1}+n_{2}+n_{3}$ can be summed up to give

$$
\frac{n(\Phi, T)}{\kappa \cdot\left(k T^{2}\right)}=\alpha+\frac{1}{2}\left(\frac{\Phi}{k T}\right)^{2}+\int_{0}^{-\frac{\Phi}{k T}} R(t) \mathrm{d} t
$$

Case 2, $\Phi \geq 0$ :

without using the separation of equation (A1), $\mathrm{n}$ can directly be written as

$$
\begin{aligned}
& n=\int_{\Phi}^{\infty} \kappa \cdot(E-\Phi) \cdot[1+\exp (-\beta E)]^{-1} \mathrm{~d} E= \\
& =\frac{\kappa}{\beta^{2}}\left\{\int_{\beta \Phi}^{\infty} \beta E \cdot[1+\exp (-\beta E)]^{-1} \mathrm{~d}(\beta E)-\beta \Phi \cdot \int_{\beta \Phi}^{\infty}[1+\exp (-\beta E)]^{-1} \mathrm{~d}(\beta E)\right\}
\end{aligned}
$$

which is immediately recognized as $\kappa(k T)^{2} \cdot h(\beta \Phi)$ so that

$$
n=\kappa(k T)^{2} \cdot h(\beta \Phi)=\kappa(k T)^{2} \cdot\left[\alpha-\int_{0}^{\beta \Phi} R(t) \mathrm{d} t\right]
$$




\section{Figures}

(a)

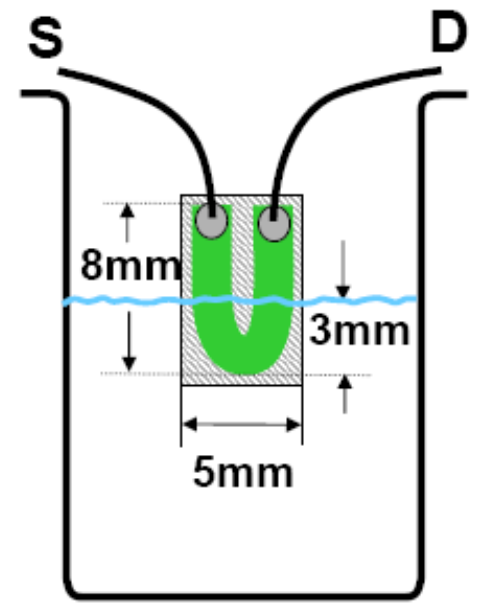

(b)

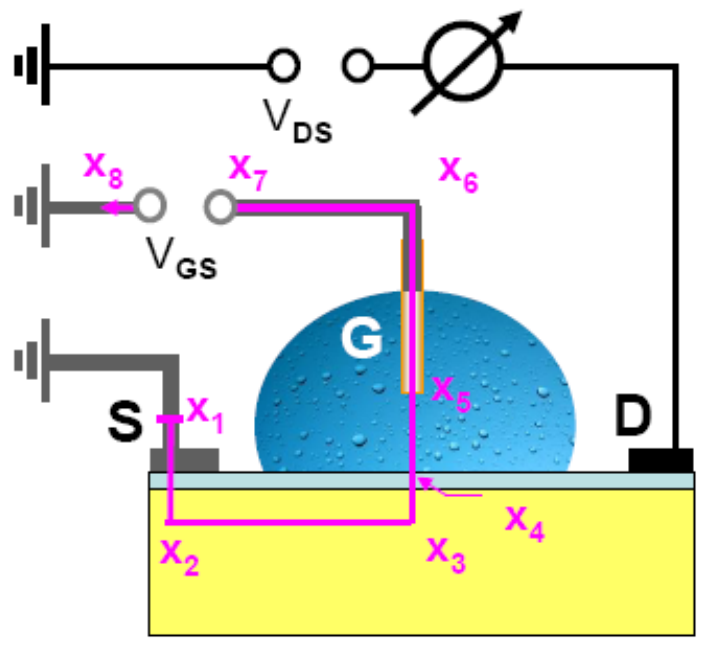

Figure 1: (Color online) Schematics of the experimental set-up (a) and the electrical circuitry (b) of the Solution Gate FET based on single layer graphene on SiC. All voltages are referenced to ground. The path from $\mathrm{x}_{1}$ to $\mathrm{x}_{8}$ is used in figure 6 .

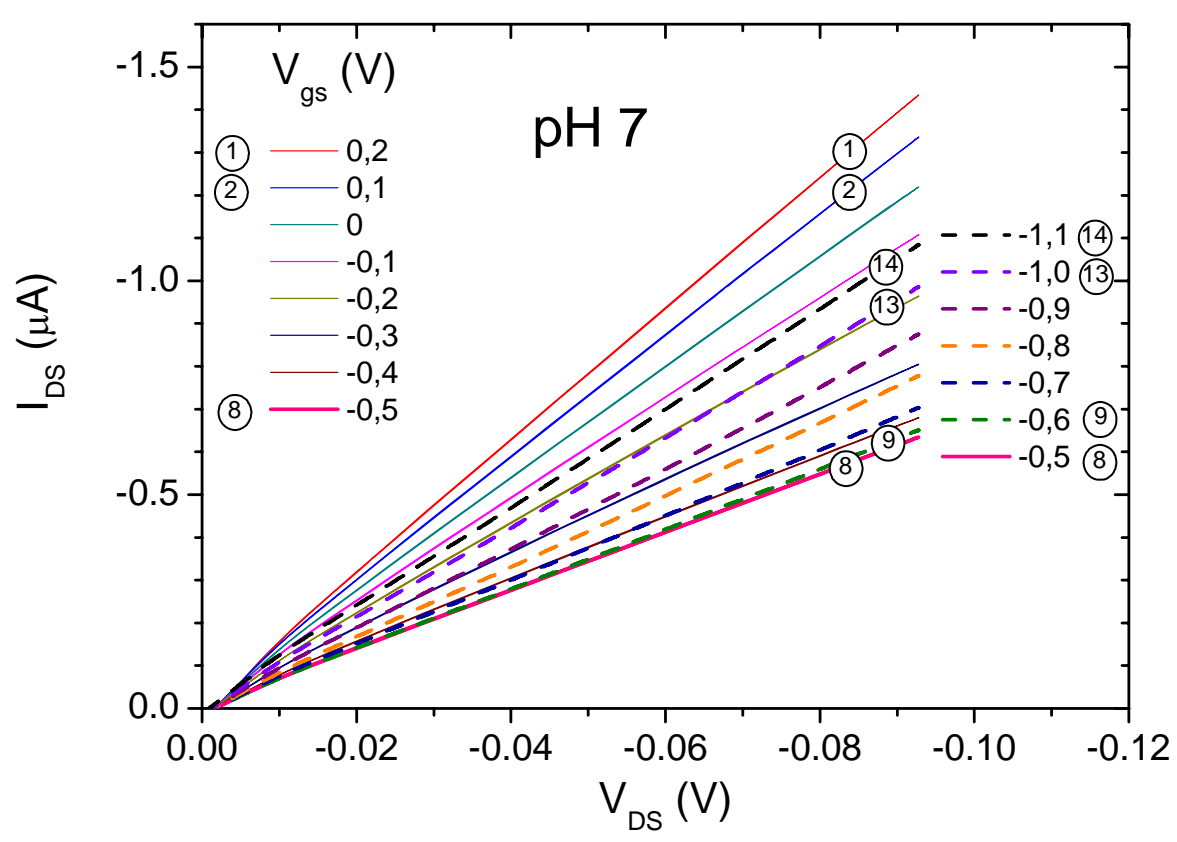

Figure 2: (Color online) Field effect output characteristics of the graphene based SGFET at a $\mathrm{pH}$ of 7. 


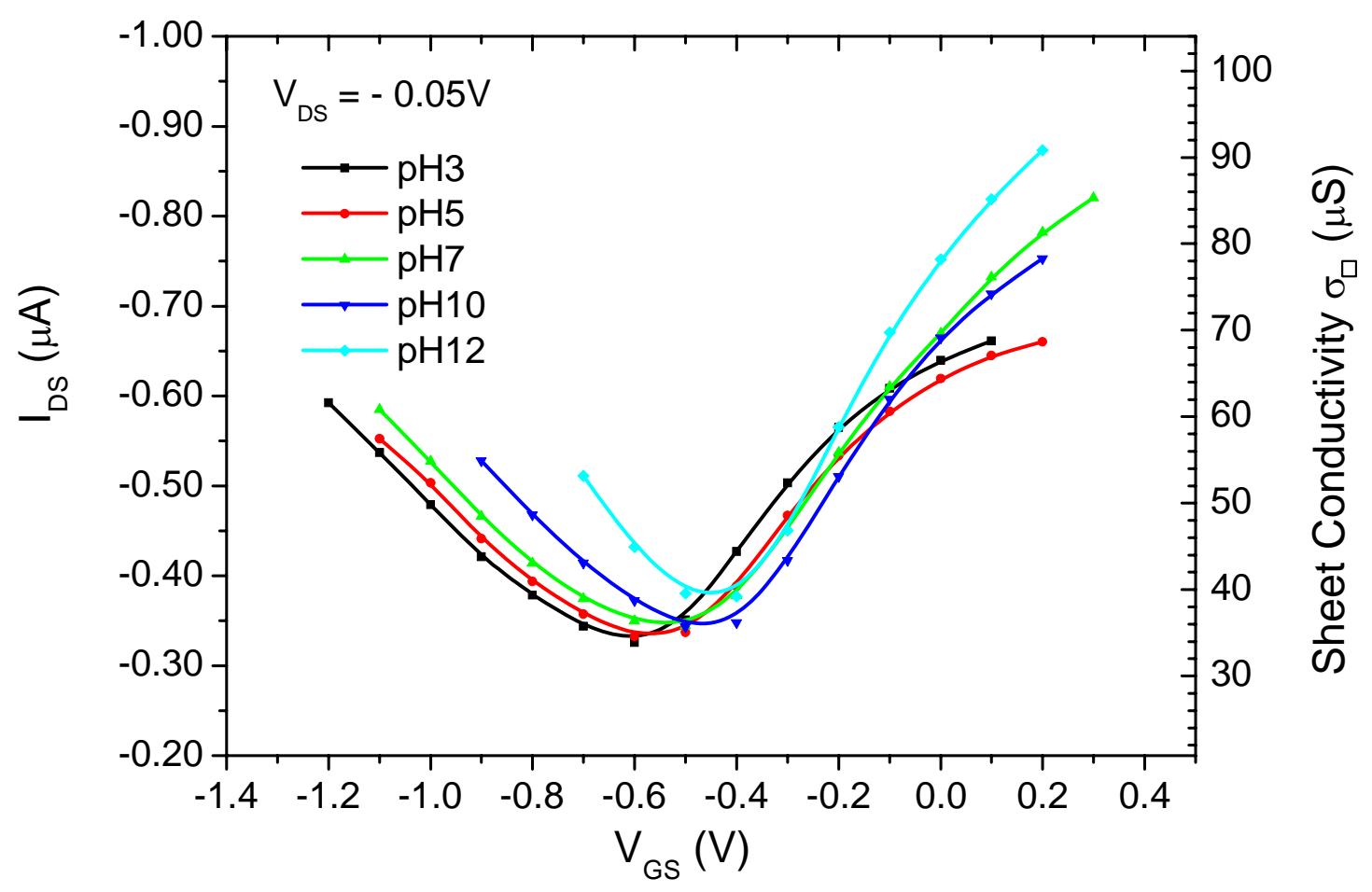

Figure 3: (Color online) Transfer characteristics of the graphene based SGFET for a constant drainsource voltage of $-0.05 \mathrm{~V}$ for different $\mathrm{pH}$ of the electrolyte.

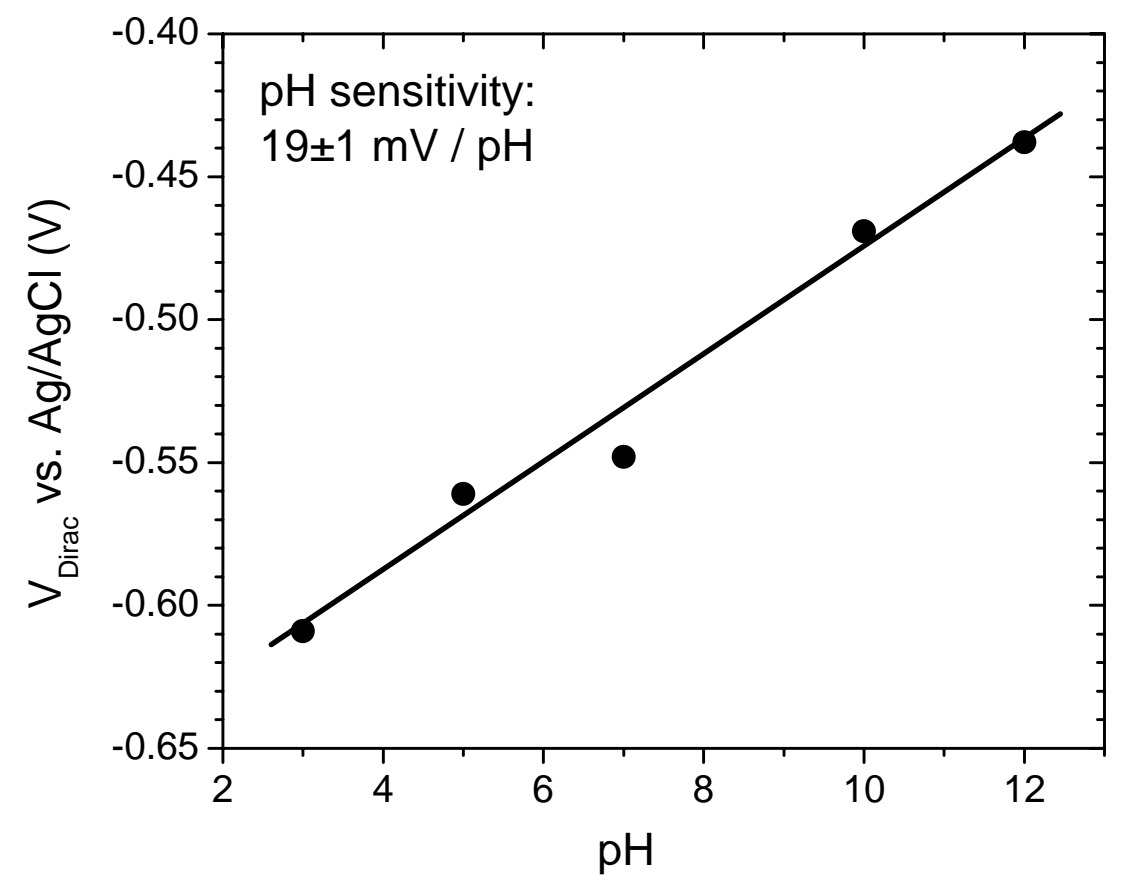

Figure 4: The $\mathrm{pH}$ sensitivity of the SGFET in the limit of small drain-source voltage $\left(V_{\mathrm{DS}}=-0.05 \mathrm{~V}\right)$ displayed as the Dirac voltage vs. $\mathrm{pH}$ with an electrochemical $\mathrm{Ag} / \mathrm{AgCl}$ reference electrode used as gate electrode. 


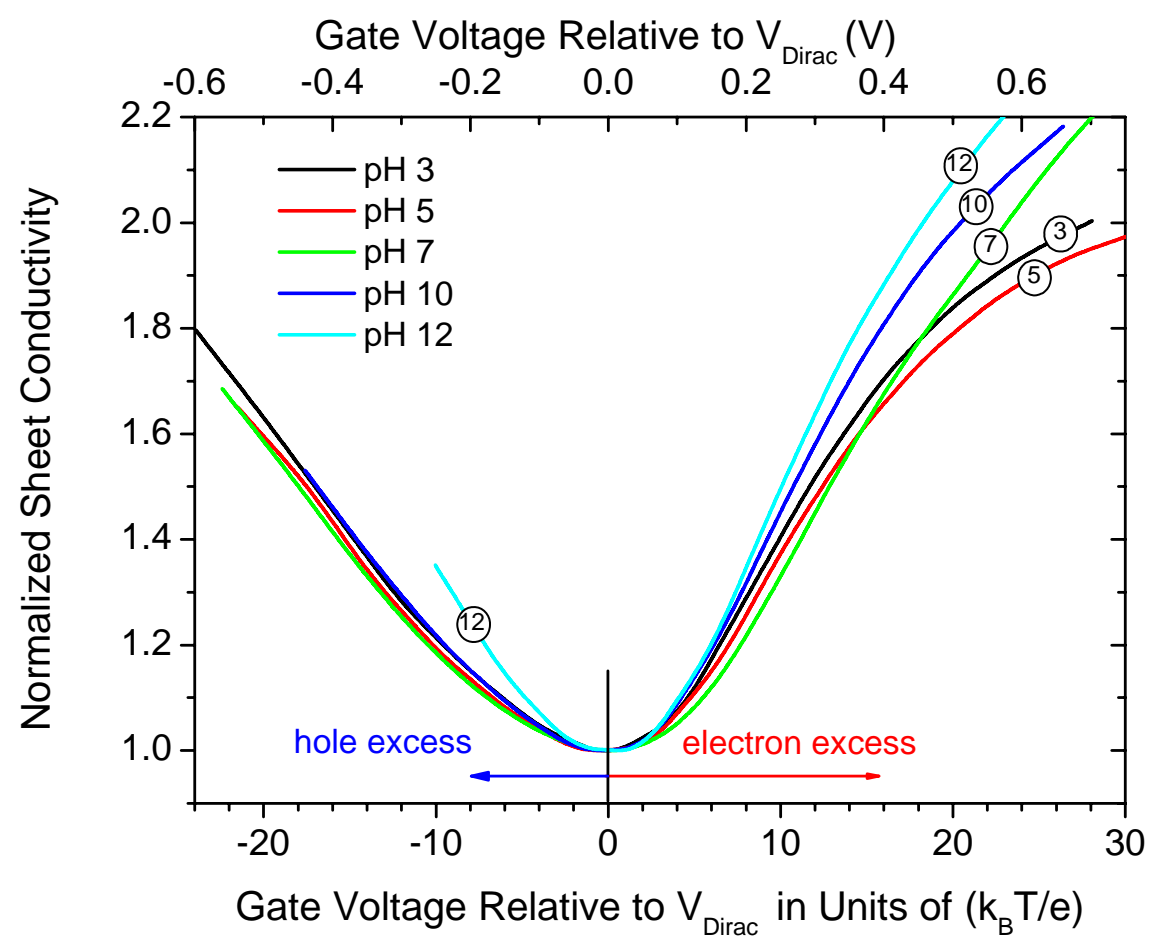

Figure 5: (Color online) Normalized plot of the transfer characteristics of the SGFET. For negative relative voltage the sheet conductivity falls on a master curve for all $\mathrm{pH}$. For the positive branch pronounced scatter of the data is observed.

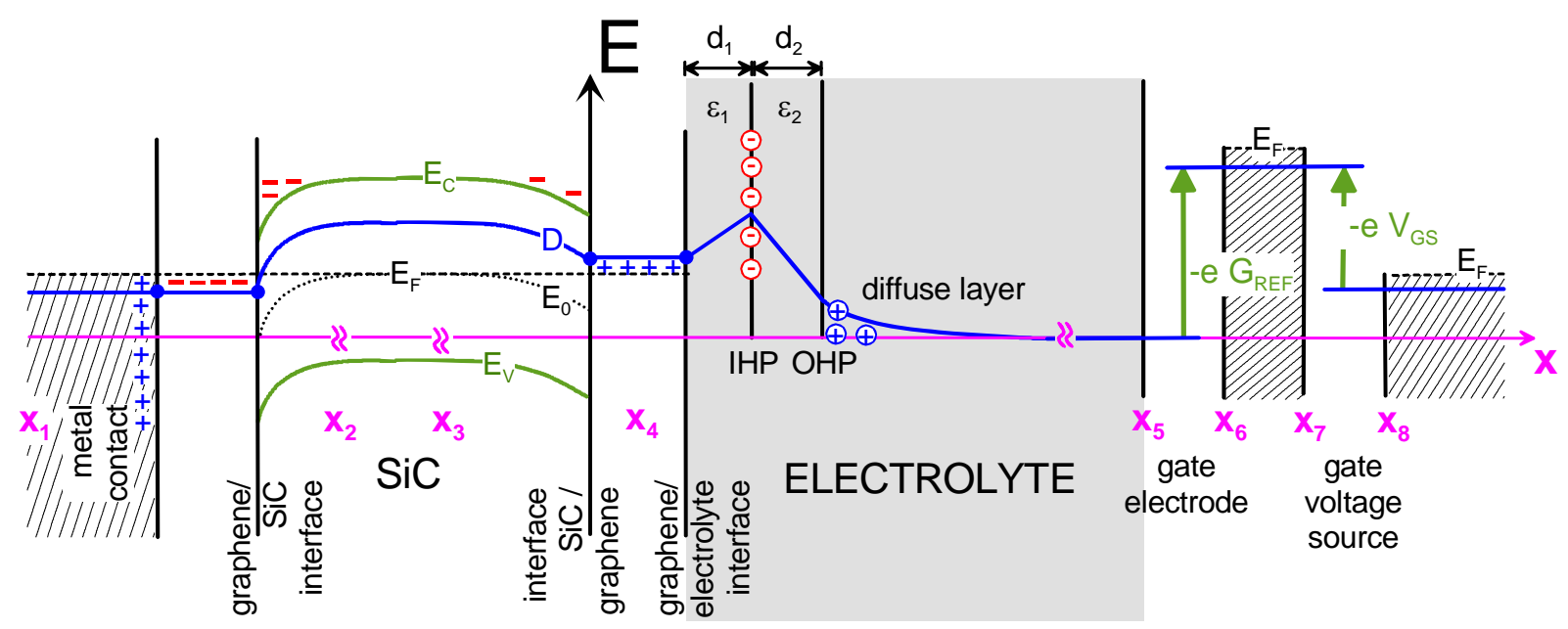

Figure 6: (Color online) The potential diagram of the complete electrical gate-source circuit of the SGFET along the path depicted in figure 1. For details see text. 


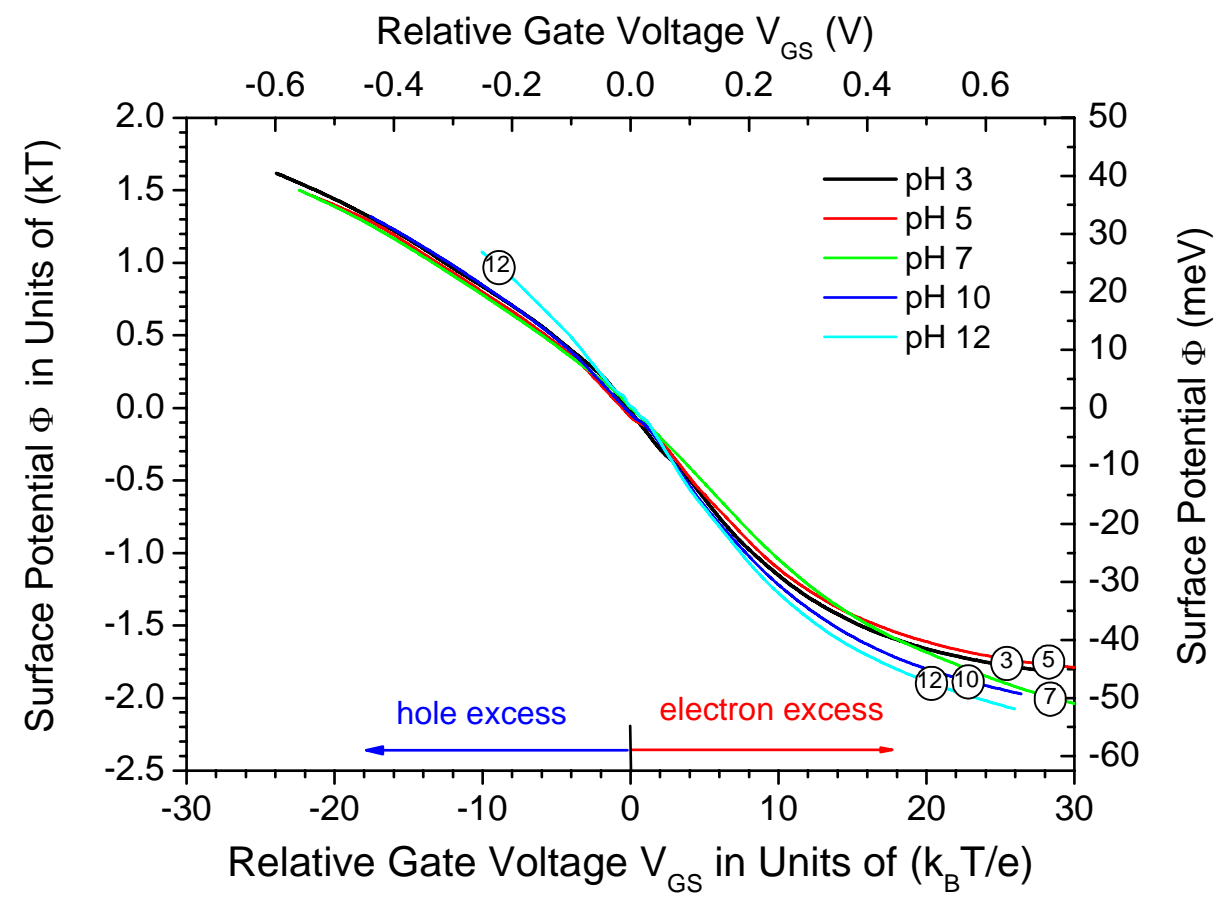

Figure 7: (Color online) Surface potential vs. relative gate voltage as extracted from figure 5 and discussed in the text.

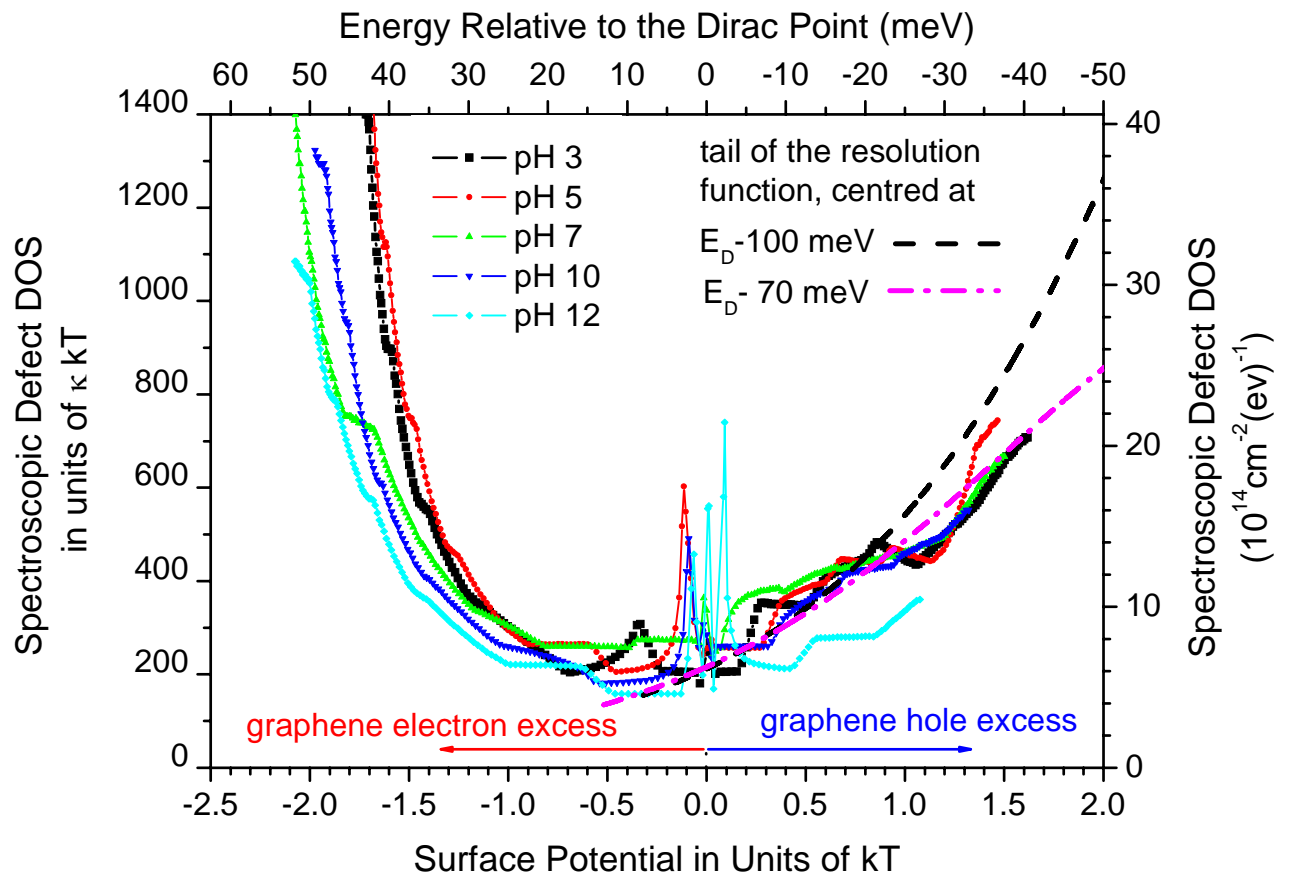

Figure 8: (Color online) Spectroscopic defect density of states for the SiC/graphene interface as evaluated from the data. The dashed and the dash-dotted lines are the tail of the resolution function for two different assumptions for the energy of interface defects relative to the Dirac energy. 


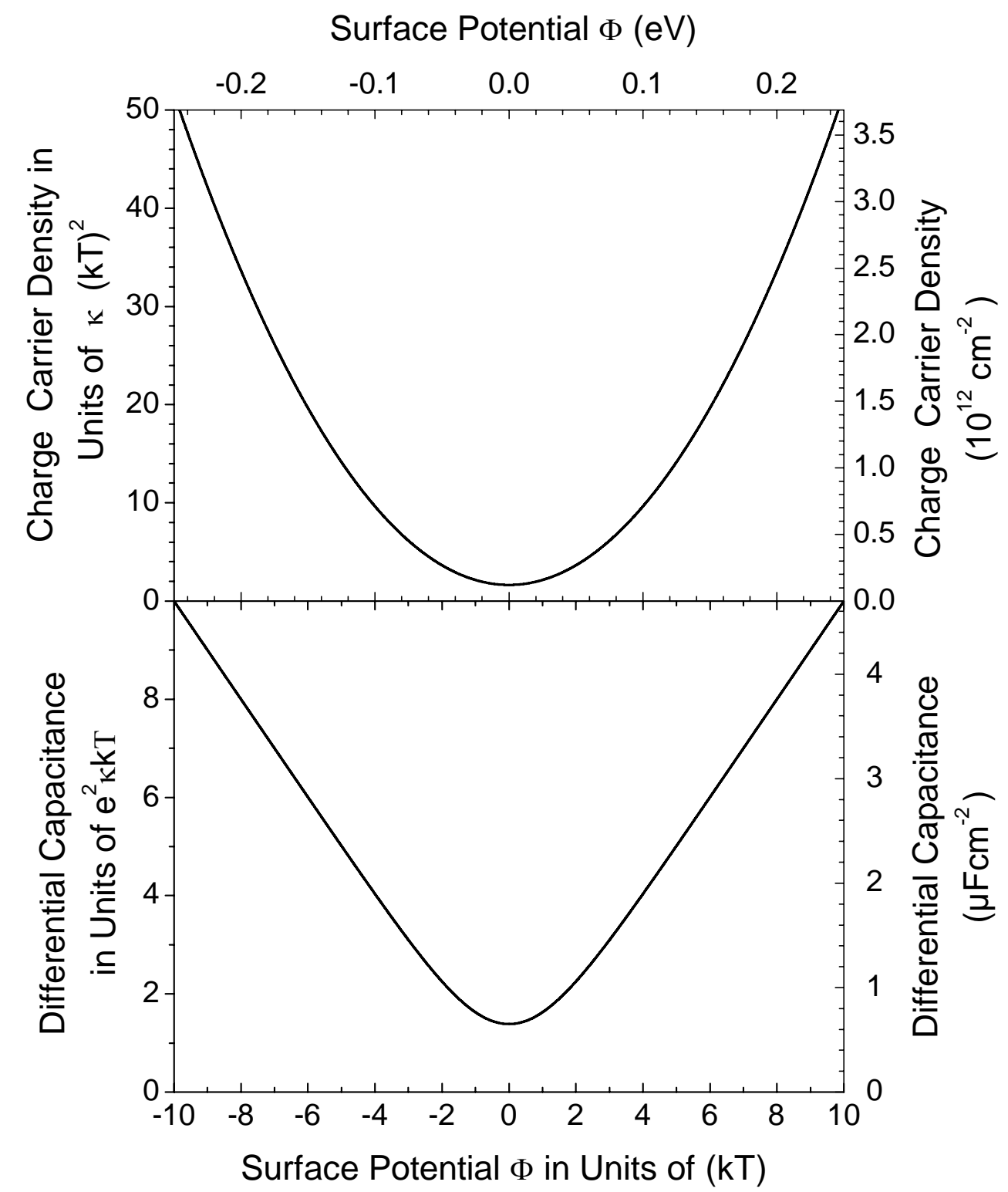

Figure 9: Temperature dependent areal charge carrier density (upper panel) and areal differential capacitance (lower panel) as a function of surface potential, i.e. of the energy difference between the Dirac energy and the Fermi level in graphene. 


\section{Figures Captions (repeated separately)}

Figure 1.: (Color online) Schematics of the experimental set-up (a) and the electrical circuitry (b) of the Solution Gate FET based on single layer graphene on $\mathrm{SiC}$. All voltages are referenced to ground. The path from $\mathrm{x}_{1}$ to $\mathrm{x}_{8}$ is used in figure 6 .

Figure 2.: (Color online) Field effect output characteristics of the graphene based SGFET at a $\mathrm{pH}$ of 7.

Figure 3.: (Color online) Transfer characteristics of the graphene based SGFET for a constant drainsource voltage of $-0.05 \mathrm{~V}$ for different $\mathrm{pH}$ of the electrolyte

Figure 4.: The $\mathrm{pH}$ sensitivity of the SGFET in the limit of small drain-source voltage $\left(\mathrm{V}_{\mathrm{DS}}=-0.05 \mathrm{~V}\right.$ ) displayed as the Dirac voltage vs. $\mathrm{pH}$ with an electrochemical $\mathrm{Ag} / \mathrm{AgCl}$ reference electrode used as gate electrode.

Figure 5.: (Color online) Normalized plot of the transfer characteristics of the SGFET. For negative relative voltage the sheet conductivity falls on a master curve for all $\mathrm{pH}$. For the positive branch pronounced scatter of the data is observed.

Figure 6.: (Color online) The potential diagram of the complete electrical gate-source circuit of the SGFET along the path depicted in figure 1. For details see text.

Figure 7.: (Color online) Surface potential vs. relative gate voltage as extracted from figure 5 and discussed in the text.

Figure 8.: (Color online) Spectroscopic defect density of states for the $\mathrm{SiC} /$ graphene interface as evaluated from the data. The dashed and the dash-dotted lines are the tail of the resolution function for two different assumptions for the energy of interface defects relative to the Dirac energy.

Figure 9.: Temperature dependent areal charge carrier density (upper panel) and areal differential capacitance (lower panel) as a function of surface potential, i.e. of the energy difference between the Dirac energy and the Fermi level in graphene. 